Original Article

\title{
CONSISE SYNTHESIS AND POTENTIAL ANTI-ANGIOGENIC ACTIVITY OF N-1 SUBSTITUTED INDOLYLCHALCONE HYBRIDS ON CHORIOALLANTOIC MEMBRANE (CAM) ASSAY
}

\author{
ANJALI M. WANEGAONKAR ${ }^{1}$, MILIND J. BHITRE${ }^{1}$, DILLIP ZAVERI ${ }^{2}$, SHRIRAM H. BAIRAGI ${ }^{3}$ \\ 1Department Pharmaceutical Chemistry C. U. Shah College of Pharmacy, S. N. D. T. Women's University, Sir Vithaldas Vidyavihar, Juhu \\ Road, Santacruz (West), Mumbai 400049, Maharashtra, India, ${ }^{2}$ Biocare Research India Pvt. Ltd., ${ }^{\text {st }}$ Floor, Kanth Complex, Above New \\ Limda Travels, Near Mahavir Jain Vidyalaya, Paldi Cross Road, Ahmedabad, ${ }^{3}$ Department Pharmaceutical Chemistry, Ideal College of \\ Pharmacy and Research, Kalyan, Maharashtra, India 421306 \\ "Email: anjaliwanegaonkar@gmail.com
}

Received: 15 Sep 2020, Revised and Accepted: 17 Nov 2020

\begin{abstract}
Objective: Synthesis of N-1 Substituted Indolylchalcone Hybrids and evaluation of anti-angiogenic activity using Chorioallantoic Membrane (CAM) Assay.

Methods: Claisen-schmidt reaction is used for the synthesis of 30 Indolylchalcone hybrids, it involves condensation of N-1 substituted indole-3carboxaldehyde and N1 substituted 2-acetyl-benzimidazole. The phase transfer catalyst, a green catalyst such as anhydrous potassium carbonate $\left(\mathrm{K}_{2} \mathrm{CO}_{3}\right)$ and PEG-400 are used in the alkylation and arylation. All synthesized indolylchalcone hybrids were evaluated for their antiangiogenic activity by in vivo-chorioallantoic membrane (CAM) assay method.
\end{abstract}

Results: The synthesized indolylchalcone compounds are evaluated. The morphometric study was carried out as described by Melkonian et al. (2002). The Compounds with code C-2, I-1, I-2 are showing the more potent effect on the dose-dependent assay of CAM. The compounds with code C-1, C-3, E-1 to E-3, M-1 and M-5 shows the significant activity, however, though the compounds with code B-1, B-2, CL-1 and A-5 were showing antiangiogenic effect at $0.1 \mu \mathrm{M}$, but does not show any significant activity on dose-dependent assay of CAM.

Conclusion: The synthesized Indolylchalcones as shown in the graph possess very good dose-dependent anti-angiogenic activities. The potency of anti-angiogenetic activity shows that methyl>Ethyl>Cl-benzyl>Benzyl>Isobutyl. 2-acetyl benzimidazole analogs have possible future scope to develop as potent angiogenesis inhibitors.

Keywords: Angiogenesis, Indole chalcone, CAM assay

(C) 2021 The Authors. Published by Innovare Academic Sciences Pvt Ltd. This is an open access article under the CC BY license (https://creativecommons.org/licenses/by/4.0/) DOI: https://dx.doi.org/10.22159/ijcpr.2021v13i1.40822. Journal homepage: https://innovareacademics.in/journals/index.php/ijcpr

\section{INTRODUCTION}

Angiogenesis or neovascularization is a combination of two Greek words, "angio" and "genesis" means vase and birth, respectively. Development of these blood capillaries, vessels plays a critical role in cell division, proliferation and movement [1]. More than $30 \mathrm{y}$ ago, the scientist Folkman proposed that the role of these blood vessel to supply food, oxygen and nutrious to malignant tumor because of that growth was dependent on the development of tumor-associated blood vessels, a process called angiogenesis [2]. Targeting angiogenesis has proven to be an effective strategy in several malignant tumour not only colorectal, gastric and lung tumour but also breast, cervical and ovary tumour [3]. Anti-vascular approaches to cancer therapy may be primarily divided into antiangiogenesis inhibitors and vascular disrupting agents (VDAs) $[4,5]$.

The well-known marketed anti-angiogenic inhibitors drug, bevacizumab (Avastin) [6], and sorafenib (Nexavar, BAY 43-9006) and sunitinib malate (Sutent, SU11248) [7] have been approved by the United States Food and Drug Administration for the treatment of cancer patients. However, these marketed drugs has serious side effects, such as hypertension, bleeding and gastrointestinal perforation; they have been associated with currently available antiVEGF agents, limiting their chronic use [8]. Hence, there is an urgent need to find a chalcone hybrid molecule that can be more potent towards cancerous cell and less toxic to normal cell but also may overcome the problem of multidrug-resistant strains.

It is well know that all type of heterocyclic ring comprises a very core active moiety or the pharmacophore [9-12]. Derivatives of nitrogen-containing fused heterocyclic compounds such as benzimidazole and indole core molecule have found number of application as an anticancer agent [13]. Literature survey reveals that there is interest in the indolylchalcone systems for use in cancer therapy and such indolylchalcone have the potential to improve the selectivity of chemotherapeutic agents and hence reduce unwanted side effects and multidrug resistance MDR syndrome [14, 15]. The well-known antiangiogenic drug sunitinib also has indole core, taking this in an account, chemistry of 3-substitution on indole moity shows that these has various biological activities and synthesis of such derivatives which has a skeleton that contains an indole cores and are also in chemistry of benzimidazole 2-substitution are valid for biological activities. Considering this in our study of rational research, we fused these indole and benzimidazole moieties with chalcone like structure (fig. 1). Thus three scaffolds moieties in our design are based on the diversity of these moieties in nature as well as in various marketed drugs.

Now a day's 2-acetylbenzimidazole molecule and indole-3carboxaldehyde are associated with a wide range of biological activities such as anticancer, anti-inflammatory, analgesic and anthelmintic etc. The $\mathrm{N}$-substituted fused heterocyclic compounds are usually biologically active and may be used as potential therapeutic alternatives to the antitumor drug.

\section{MATERIALS AND METHODS}

\section{Synthesis of indolylchalcone}

In this research study, a series of 30 Indolylchalcone hybrids were synthesized by Claisen-schmidt condensation of $\mathrm{N}-1$ substituted indole-3-carboxaldehyde and N1 substituted 2-acetylbenzimidazole. The reaction involved in the synthesis of indolylchalcone hybrids as shown in (Scheme 1). The $\mathrm{N}$ alkylation/arylation, mono-methylation on indole-3-carboxaldehyde were achieved by using alkylating and arylating agents in the 
presence of phase transfer catalyst, a green catalyst such as anhydrous potassium carbonate $\left(\mathrm{K}_{2} \mathrm{CO}_{3}\right)$ and PEG-400. The $\mathrm{N} 1$ substitution reaction on indole-3-carboxaldehyde and 2-aceylbenzimidazole as shown in (scheme $1 \mathrm{~A}$ and scheme 1B). The physicochemical properties and analytical data of synthesized indolylchalcone compounds are mentioned in table 1 and 2 , respectively.<smiles>[R7]c1c(C=O)c2ccccc2n1[PH3+]</smiles>

substituted derivatives $3 \mathrm{H}$-indole-3-carbaldehyde

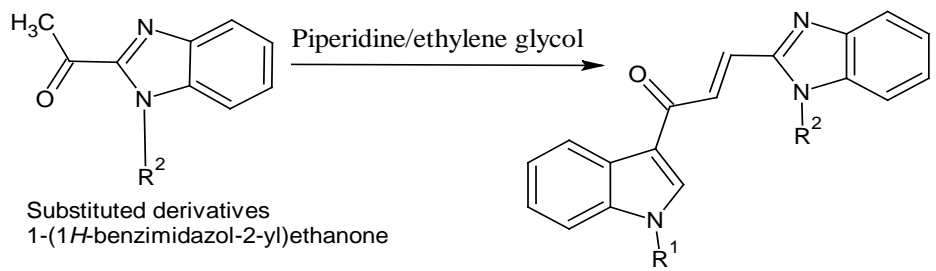

Chalcones of indole derivatives

$\mathrm{R}=$ Benzyl, Ethyl, Allyl etc

Scheme 1: Synthesis of indole chalcone<smiles>[R]c1[nH]c2ccccc2c1C=O</smiles>

Scheme 1A: Synthesis of N-substituted indole-3-carbaldehyde derivatives

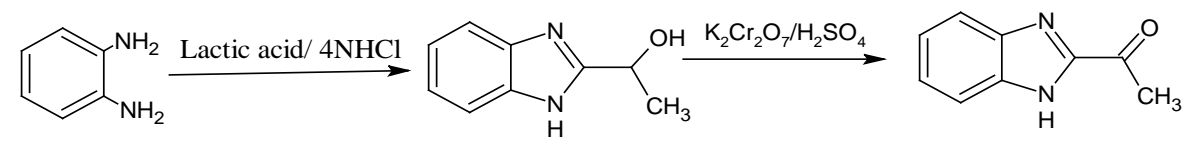

benzene-1,2-diamine

1-(1H-benzimidazol-2-yl)ethanol

2-acetyl benzimidazole

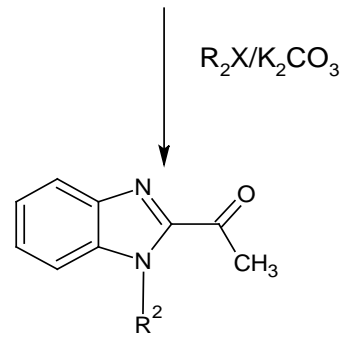

N1derivatives 2- acetyl benzimidazole

Scheme 1B: Synthesis of N-derivatives of 2-acetyl benzimidazole

\section{Anti-angiogenic activity}

\section{Chicken egg chorioallantoic membrane assay (CAM)}

The fertile eggs of $6^{\text {th }}$ day was procured from IPDP poultry Maqarba, Ahmedabad and Angiogenesis inhibitory activities of 30 indolylchalcone hybrids were evaluated using quantitative CAM assay [16-18], Out of which 15 Indolylchalcone hybrids shows most inhibitory effect on in vivo assay models of angiogenesis as shown in (table 3). The morphometric study was carried out as described by Melkonian et al. (2002). Further, these indolylchalcone hybrids also shows the dose-dependent percentage inhibition of antiangiogenic activity of tested compounds calculated as per given formula (fig. 1)
$\%$ inhibition $=[($ vessel number of untreated CAM-vessel number of CAM treated with test compounds)/vessel number of untreated CAM] x100

\section{RESULTS}

The Compounds with code C-2, I- $1, \mathrm{I}-2$ are showing more potent effect on dose dependent assay of CAM when compared to standard. Compounds with code C-2 and I-2 consist of N-1 substitution on ethyl group on both the indole and benzimidazole. Compound with code I- 1 is unsubstituted indole, which consists of $\mathrm{N}-1$ substitution of iso-butyl moity on benzimidazole. The compounds with code $\mathrm{C}-1$, C-3, E- 1 to E-3, M-1 and M-5 shows the significant activity, however, though the compounds with code B-1, B-2, CL-1 and A-5 were showing antiangiogenic effect at $0.1 \mu \mathrm{M}$, but does not show any significant activity on dose-dependent assay of CAM. 
Table 1: Physicochemical properties of synthesized indolylchalcone

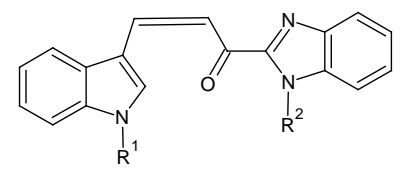

\begin{tabular}{|c|c|c|c|c|c|c|c|c|}
\hline $\begin{array}{l}\text { S. } \\
\text { No. }\end{array}$ & $\begin{array}{l}\text { Name of the } \\
\text { compound }\end{array}$ & R-1 & R-2 & $\begin{array}{l}\text { Molecular } \\
\text { weight }\end{array}$ & $\begin{array}{l}\text { Molecular } \\
\text { formula }\end{array}$ & $\begin{array}{l}\% \text { yield of } \\
\text { chalcone reaction }\end{array}$ & $\begin{array}{l}\text { Melting } \\
\text { point }\end{array}$ & $\mathbf{R F}$ \\
\hline 1. & $\mathrm{C}-1$ & $\mathrm{H}$ & $\mathrm{H}$ & 287.31532 & $\mathrm{C}_{18} \mathrm{H}_{13} \mathrm{~N}_{3} \mathrm{O}$ & 77 & $265^{\circ} \mathrm{C}$ & 0.6123 \\
\hline 2. & $\mathrm{C}-2$ & Ethyl & $\mathrm{H}$ & 315.36848 & $\mathrm{C}_{20} \mathrm{H}_{17} \mathrm{~N}_{3} \mathrm{O}$ & 65 & $213^{\circ} \mathrm{C}$ & 0.1711 \\
\hline 3. & $\mathrm{C}-3$ & Benzyl & $\mathrm{H}$ & 377.43786 & $\mathrm{C}_{25} \mathrm{H}_{19} \mathrm{~N}_{3} \mathrm{O}$ & 68 & $249^{\circ} \mathrm{C}$ & 0.45234 \\
\hline 4. & $C-4$ & 2-Cl benzyl & $\mathrm{H}$ & 411.8892 & $\mathrm{C}_{25} \mathrm{H}_{18} \mathrm{ClN}_{3} \mathrm{O}$ & 85 & $223^{\circ} \mathrm{C}$ & 0.2105 \\
\hline 5. & B-1 & $\mathrm{H}$ & Benzyl & 377.4376 & $\mathrm{C}_{25} \mathrm{H}_{19} \mathrm{~N}_{3} \mathrm{O}$ & 70 & $139^{\circ} \mathrm{C}$ & 0.2630 \\
\hline 6. & B-2 & Ethyl & Benzyl & 405.49102 & $\mathrm{C}_{27} \mathrm{H}_{23} \mathrm{~N}_{3} \mathrm{O}$ & 69 & $155^{\circ} \mathrm{C}$ & 0.756 \\
\hline 7. & B-3 & Benzyl & Benzyl & 467.5604 & $\mathrm{C}_{32} \mathrm{H}_{25} \mathrm{~N}_{3} \mathrm{O}$ & 72 & $183^{\circ} \mathrm{C}$ & 0.842 \\
\hline 8. & B-4 & 2-Cl benzyl & Benzyl & 502.00546 & $\mathrm{C}_{32} \mathrm{H}_{24} \mathrm{ClN}_{3} \mathrm{O}$ & 75 & $171^{\circ} \mathrm{C}$ & 0.754 \\
\hline 9. & E-1 & $\mathrm{H}$ & Ethyl & 315.36898 & $\mathrm{C}_{20} \mathrm{H}_{17} \mathrm{~N}_{3} \mathrm{O}$ & 55 & $205^{\circ} \mathrm{C}$ & 0.641 \\
\hline 10. & E-2 & Ethyl & Ethyl & 343.42164 & $\mathrm{C}_{22} \mathrm{H}_{21} \mathrm{~N}_{3} \mathrm{O}$ & 60 & $177^{\circ} \mathrm{C}$ & 0.6728 \\
\hline 11. & E-3 & Benzyl & Ethyl & 405.49102 & $\mathrm{C}_{27} \mathrm{H}_{23} \mathrm{~N}_{3} \mathrm{O}$ & 65 & $216^{\circ} \mathrm{C}$ & 0.5553 \\
\hline 12. & E-4 & 2-Cl benzyl & Ethyl & 439.93608 & $\mathrm{C}_{27} \mathrm{H}_{22} \mathrm{ClN}_{3} \mathrm{O}$ & 77 & $149^{\circ} \mathrm{C}$ & 0.453 \\
\hline 13. & CL-1 & $\mathrm{H}$ & 2-Cl benzyl & 411.8892 & $\mathrm{C}_{25} \mathrm{H}_{18} \mathrm{ClN}_{3} \mathrm{O}$ & 79 & $219^{\circ} \mathrm{C}$ & 0.2156 \\
\hline 14. & CL-2 & Ethyl & 2-Cl benzyl & 439.9360 & $\mathrm{C}_{27} \mathrm{H}_{22} \mathrm{ClN}_{3} \mathrm{O}$ & 81 & $181^{\circ} \mathrm{C}$ & 0.7800 \\
\hline 15. & CL-3 & Benzyl & 2-Cl benzyl & 502.00546 & $\mathrm{C}_{32} \mathrm{H}_{24} \mathrm{ClN}_{3} \mathrm{O}$ & 84 & $232^{\circ} \mathrm{C}$ & 0.8600 \\
\hline 16. & CL-4 & 2-Cl benzyl & 2-Cl benzyl & 536.45052 & $\mathrm{C}_{32} \mathrm{H}_{23} \mathrm{Cl}_{2} \mathrm{~N}_{3} \mathrm{O}$ & 68 & $226^{\circ} \mathrm{C}$ & 0.8039 \\
\hline 17. & $A-1$ & Allyl & $\mathrm{H}$ & 327.37918 & $\mathrm{C}_{21} \mathrm{H}_{19} \mathrm{~N}_{3} \mathrm{O}$ & 65 & $122^{\circ} \mathrm{C}$ & 0.1428 \\
\hline 18. & A-2 & Allyl & Ethyl & 355.43234 & $\mathrm{C}_{23} \mathrm{H}_{23} \mathrm{~N}_{3} \mathrm{O}$ & 68 & $101^{\circ} \mathrm{C}$ & 0.3564 \\
\hline 19. & A-3 & Allyl & Benzyl & 417.50172 & $\mathrm{C}_{28} \mathrm{H}_{25} \mathrm{~N}_{3} \mathrm{O}$ & 71 & $132^{\circ} \mathrm{C}$ & 0.5870 \\
\hline 20. & $A-4$ & Allyl & 2-Cl benzyl & 451.94678 & $\mathrm{C}_{28} \mathrm{H}_{24} \mathrm{ClN}_{3} \mathrm{O}$ & 60 & $139^{\circ} \mathrm{C}$ & 0.5612 \\
\hline 21. & $A-5$ & Allyl & Iso-Butyl & 399.5279 & $\mathrm{C}_{25} \mathrm{H}_{27} \mathrm{~N}_{3} \mathrm{O}$ & 70 & $95^{\circ} \mathrm{C}$ & 0.4012 \\
\hline 22. & M-1 & Methyl & $\mathrm{H}$ & 301.3419 & $\mathrm{C}_{19} \mathrm{H}_{15} \mathrm{~N}_{3} \mathrm{O}$ & 71 & $142^{\circ} \mathrm{C}$ & 0.1451 \\
\hline 23. & M-2 & Methyl & Ethyl & 329.39506 & $\mathrm{C}_{21} \mathrm{H}_{19} \mathrm{~N}_{3} \mathrm{O}$ & 65 & $224^{\circ} \mathrm{C}$ & 0.4166 \\
\hline 24. & M-3 & Methyl & Benzyl & 391.46444 & $\mathrm{C}_{26} \mathrm{H}_{21} \mathrm{~N}_{3} \mathrm{O}$ & 66 & $231^{\circ} \mathrm{C}$ & 0.4833 \\
\hline 25. & M-4 & Methyl & 2-Cl benzyl & 425.9095 & $\mathrm{C}_{26} \mathrm{H}_{20} \mathrm{ClN}_{3} \mathrm{O}$ & 80 & $220^{\circ} \mathrm{C}$ & 0.6353 \\
\hline 26. & M-5 & Methyl & Iso-Butyl & 357.4422 & $\mathrm{C}_{23} \mathrm{H}_{23} \mathrm{~N}_{3} \mathrm{O}$ & 79 & $141^{\circ} \mathrm{C}$ & 0.5833 \\
\hline 27. & $\mathrm{I}-1$ & $\mathrm{H}$ & Iso-butyl & 343.42164 & $\mathrm{C}_{22} \mathrm{H}_{21} \mathrm{~N}_{3} \mathrm{O}$ & 65 & $131^{\circ} \mathrm{C}$ & 0.6857 \\
\hline 28. & $\mathrm{I}-2$ & Ethyl & Iso-butyl & 371.4748 & $\mathrm{C}_{24} \mathrm{H}_{25} \mathrm{~N}_{3} \mathrm{O}$ & 70 & $116^{\circ} \mathrm{C}$ & 0.5421 \\
\hline 29. & $\mathrm{I}-3$ & Benzyl & Iso-butyl & 433.54418 & $\mathrm{C}_{29} \mathrm{H}_{27} \mathrm{~N}_{3} \mathrm{O}$ & 73 & $129^{\circ} \mathrm{C}$ & 0.5483 \\
\hline 30. & $\mathrm{I}-4$ & 2-Cl benzyl & Iso-butyl & 467.94678 & $\mathrm{C}_{29} \mathrm{H}_{26} \mathrm{ClN}_{3} \mathrm{O}$ & 68 & $125^{\circ} \mathrm{C}$ & 0.6140 \\
\hline
\end{tabular}

Table 2: Analytical data of synthesized indolylchalcone

\begin{tabular}{|c|c|c|c|}
\hline $\begin{array}{l}\text { S. } \\
\text { No. }\end{array}$ & $\begin{array}{l}\text { Name of the } \\
\text { compound }\end{array}$ & Wavenumber $\left(\mathrm{cm}^{-1}\right)$ & Chemical shift ( $\delta$ ppm) \\
\hline 1. & $\mathrm{C}-1$ & $\begin{array}{l}\text { 3424, 3226(NH str), } 1639(\mathrm{C}=0), 1562(\mathrm{CH}=\mathrm{CH} \\
\text { Str), }\end{array}$ & $\begin{array}{l}\text { 7.26-7.30 (m, 4H, Ar-CH),7.5 (d, H, C-O-CH), 7.7(br, 2H, NH), } 8.05(\mathrm{t}, 3 \mathrm{H} \text {, } \\
\text { Ar) } 8.26(\mathrm{~d}, 1 \mathrm{H} \mathrm{CH}=\mathrm{CH}), 8.29(\mathrm{~d}, 2 \mathrm{H}, \mathrm{Ar}) \mathrm{ppm} .\end{array}$ \\
\hline 2. & $\mathrm{C}-2$ & $\begin{array}{l}3224(\mathrm{NH} \text { Str }) 3055,2934\left(\mathrm{CH}_{2} \mathrm{CH}_{3}\right) 2978(- \\
\left.\mathrm{CH}_{3}-\mathrm{Str}\right) 1646(\mathrm{C}=0) 1523(\mathrm{C}=\mathrm{H} \mathrm{Str})\end{array}$ & $\begin{array}{l}1.55\left(\mathrm{~s}, 3 \mathrm{H}, \mathrm{CH}_{3}\right) 4.24\left(\mathrm{~m}, 2 \mathrm{H}, \mathrm{CH}_{2}\right) 7.75(\mathrm{~d}, \mathrm{H}, \mathrm{C}-\mathrm{O}-\mathrm{CH}) 7.29-7.33(\mathrm{~m}, 4 \mathrm{H}, \\
\mathrm{Ar}-\mathrm{CH}), 7.96(\mathrm{~d}, 1 \mathrm{H}, \mathrm{CH}=\mathrm{CH}), 8.13(\mathrm{t}, 3 \mathrm{H}, \mathrm{Ar}-\mathrm{CH}), 8.42(\mathrm{~d}, 2 \mathrm{H}, \mathrm{Ar}-\mathrm{CH}) \\
10.71(\mathrm{br}, 1 \mathrm{H}, \mathrm{NH}) \mathrm{ppm}\end{array}$ \\
\hline 3. & $\mathrm{C}-3$ & $\begin{array}{l}3258(\mathrm{NH}-\mathrm{str}), 3100,3058,(\mathrm{CH}- \\
\text { str,Ar),1648(C=0),1528 } \\
\text { (C=H Str), }\end{array}$ & $\begin{array}{l}5.50\left(\mathrm{~s}, 2 \mathrm{H}, \mathrm{CH}_{2}\right), 7.51(\mathrm{~d}, \mathrm{H}, \mathrm{C}-\mathrm{O}-\mathrm{CH}), 7.25-7.36(\mathrm{~m}, 8 \mathrm{H}, \mathrm{Ar}-\mathrm{CH}), 7.81(\mathrm{~d}, \\
1 \mathrm{H}, \mathrm{CH}=\mathrm{CH}), 8.06(\mathrm{t}, 3 \mathrm{H}, \mathrm{Ar}-\mathrm{CH}), 8.23(\mathrm{~d}, 2 \mathrm{H}, \mathrm{Ar}-\mathrm{CH}), 13.24(\mathrm{br}, 1 \mathrm{H}, \\
\mathrm{NH}) \mathrm{ppm} .\end{array}$ \\
\hline 4. & $\mathrm{C}-4$ & $\begin{array}{l}\text { 3248(NH-Str), 3120,3058(CH str, Ar),1658 } \\
(\mathrm{C}=0), 1525(\mathrm{C}=\mathrm{H} \mathrm{Str}), 737(\mathrm{C}-\mathrm{Cl})\end{array}$ & $\begin{array}{l}\left.\text { 5.57(s, 2H, } \mathrm{CH}_{2}\right), 7.85(\mathrm{~d}, 1 \mathrm{H}, \mathrm{CH}=\mathrm{CH}) 7.51(\mathrm{~d}, 1 \mathrm{H}, \mathrm{C}-0-\mathrm{CH}), 7.19-7.33(\mathrm{~m}, \\
8 \mathrm{H}, \mathrm{Ar}-\mathrm{CH}), 8.10(\mathrm{~m}, 4 \mathrm{H}, \mathrm{Ar}-\mathrm{CH}) 13.1(\mathrm{br}, 1 \mathrm{H}, \mathrm{NH}) \mathrm{ppm} .\end{array}$ \\
\hline 5. & B-1 & $\begin{array}{l}3208(\mathrm{NH} \text { str }), 3068(\mathrm{CH} \text { str, } \mathrm{Ar}), 1650(\mathrm{C}=0) \\
1530(\mathrm{CH}=\mathrm{CH} \text { Str }), 1477(\mathrm{C}=\mathrm{N} \text { strAr })\end{array}$ & $\begin{array}{l}3.46\left(\mathrm{~s}, 2 \mathrm{H}, \mathrm{CH}_{2}\right), 6.03(\mathrm{~d}, 1 \mathrm{H}, \mathrm{C}-\mathrm{O}-\mathrm{CH}), 7.77,(\mathrm{~d}, 1 \mathrm{H}, \mathrm{CH}=\mathrm{CH}) 7.19 \text { to } 7.77 \\
(\mathrm{~m}, 8 \mathrm{H}, \mathrm{Ar}-\mathrm{CH}), 8.16(\mathrm{t}, 3 \mathrm{H}, \mathrm{Ar}), 8.31(\mathrm{~d}, 2 \mathrm{H}, \mathrm{Ar}-\mathrm{CH}), 12.87(\mathrm{br}, 1 \mathrm{H}, \mathrm{NH}) \mathrm{ppm} .\end{array}$ \\
\hline 6. & B-2 & $\begin{array}{l}3059,3032,3007(\mathrm{CH} \mathrm{str}, \mathrm{Ar}) 2980\left(\mathrm{CH}_{2} \mathrm{CH}_{3}\right) \\
1650(\mathrm{C}=0), 1525(\mathrm{CH}=\mathrm{CH} \text { Str })\end{array}$ & $\begin{array}{l}1.50\left(\mathrm{t}, 3 \mathrm{H}, \mathrm{CH}_{3}\right) 4.18\left(\mathrm{q}, 2 \mathrm{H}, \mathrm{CH}_{2}\right), 6.06\left(\mathrm{~s}, 2 \mathrm{H}, \mathrm{Ar}-\mathrm{CH}_{2}\right) \mathrm{7.21}(\mathrm{d}, 1 \mathrm{H}, \mathrm{C}-\mathrm{O}-\mathrm{CH}) \\
7.60(\mathrm{~d}, 1 \mathrm{H}, \mathrm{CH}=\mathrm{CH}), 7.23-7.36(\mathrm{~m}, 8 \mathrm{H}, \mathrm{Ar}-\mathrm{CH}) 7.85(\mathrm{t}, 3 \mathrm{H}, \mathrm{Ar}-\mathrm{CH}), \\
7.96(\mathrm{~d}, 2 \mathrm{H}, \mathrm{Ar}-\mathrm{CH}) \mathrm{ppm} .\end{array}$ \\
\hline 7. & B-3 & $\begin{array}{l}\text { 3061,3031, (CH str, Ar) 2927 }\left(-\mathrm{CH}_{2}\right) \\
1651(\mathrm{C}=0) \text { 1525(CH=CH Str })\end{array}$ & $\begin{array}{l}\left.\text { 5.47(s, 2H, } \mathrm{CH}_{2}-\mathrm{Ar}\right), 5.71\left(\mathrm{~s}, 2 \mathrm{H}, \mathrm{CH}_{2}-\mathrm{Ar}\right), 6.32(\mathrm{~d}, \mathrm{H}, \mathrm{C}-\mathrm{O}-\mathrm{CH}), 7.21(\mathrm{~d}, 1 \mathrm{H}, \\
\mathrm{CH}=\mathrm{CH}), 6.39 \text { to } 7.50(\mathrm{~m}, 13 \mathrm{H}, \mathrm{Ar}-\mathrm{CH}), 8.04(\mathrm{~m}, 5 \mathrm{H}, \mathrm{Ar}-\mathrm{CH}) \mathrm{ppm} .\end{array}$ \\
\hline 8. & B-4 & $\begin{array}{l}3061,3031(\mathrm{CHAr}-\mathrm{str},), 2927\left(\mathrm{CH}_{2} \mathrm{Str}\right) \\
1651(\mathrm{C}=0), 1525(\mathrm{CH}=\mathrm{CH} \text { str }), 623(\mathrm{C}-\mathrm{Cl})\end{array}$ & $\begin{array}{l}\left.\text { 5.45(s, 2H, } \mathrm{CH}_{2}-\mathrm{Ar}\right), 6.00\left(\mathrm{~s}, 2 \mathrm{H}, \mathrm{CH}_{2}-\mathrm{Ar}\right), 6.75(\mathrm{~d}, 1 \mathrm{H}, \mathrm{C}-\mathrm{O}-\mathrm{CH}), 7.21(\mathrm{~d}, 1 \mathrm{H}, \\
\mathrm{CH}=\mathrm{CH}), 7.24-7.63(\mathrm{~m}, 14 \mathrm{H}, \mathrm{Ar}-\mathrm{CH}), 7.90-8.21(\mathrm{~m}, 4 \mathrm{H}, \mathrm{Ar}-\mathrm{CH}) \mathrm{ppm} .\end{array}$ \\
\hline 9. & E-1 & $\begin{array}{l}3229(\mathrm{NH} \text { str }), 2961\left(\mathrm{CH}_{2} \mathrm{CH}_{3}\right) \\
1638(\mathrm{C}=0), 1433(\mathrm{C}=\mathrm{C} \text { strAr })\end{array}$ & $\begin{array}{l}1.38\left(\mathrm{~s}, 3 \mathrm{H}, \mathrm{CH}_{3}\right) 4.70\left(\mathrm{q}, 2 \mathrm{H}, \mathrm{CH}_{2}\right), 7.40(\mathrm{~d}, \mathrm{H}, \mathrm{C}-\mathrm{O}-\mathrm{CH}), 7.26-7.41(\mathrm{~m}, 4 \mathrm{H}, \\
\mathrm{Ar}-\mathrm{CH}), 7.50(\mathrm{~d}, 1 \mathrm{H}, \mathrm{CH}=\mathrm{CH}), 7.89(\mathrm{~d}, 2 \mathrm{H}, \mathrm{Ar}-\mathrm{CH}), 8.12(\mathrm{t}, 3 \mathrm{H}, \mathrm{Ar}-\mathrm{CH}) \\
11.82(\mathrm{br}, 1 \mathrm{H}, \mathrm{NH}) \text { ppm. }\end{array}$ \\
\hline 10. & $\mathrm{E}-2$ & $\begin{array}{l}\text { 3053( } \mathrm{CH} \text { str, } \mathrm{Ar}), 2934\left(\mathrm{CH}_{2} \mathrm{CH}_{3}\right) \\
2978\left(\mathrm{CH}_{2} \mathrm{CH}_{3}\right), 1650(\mathrm{C}=0), 1524(\mathrm{CH}=\mathrm{CH} \mathrm{Str}) .\end{array}$ & $\begin{array}{l}1.45\left(\mathrm{t}, 3 \mathrm{H}, \mathrm{CH}_{3}\right), 1.52\left(\mathrm{t}, 3 \mathrm{H}, \mathrm{CH}_{3}\right), 4.18\left(\mathrm{q}, 2 \mathrm{H}, \mathrm{CH}_{2}\right), 4.76\left(\mathrm{q}, 2 \mathrm{H}, \mathrm{CH}_{2}\right) \\
\text { 7.65(d, } 1 \mathrm{H}, \mathrm{C}-\mathrm{O}-\mathrm{CH}), 7.96(\mathrm{~d}, \mathrm{H}, \mathrm{CH}=\mathrm{CH}), 7.25-7.45(\mathrm{~m}, 5 \mathrm{H}, \mathrm{Ar}-\mathrm{CH}) \\
\text { 8.21(t, 3H, Ar-CH) ppm. }\end{array}$ \\
\hline 11. & E-3 & $\begin{array}{l}\text { 3090, 3059, (CH str, } \mathrm{Ar}), 2980\left(\mathrm{CH}_{2} \mathrm{CH}_{3}\right) \\
1736(\mathrm{CH}-\mathrm{C}-\mathrm{O}), 1651(\mathrm{C}=0), 1569,1582 \\
(\mathrm{CH}=\mathrm{CH})\end{array}$ & $\begin{array}{l}1.49\left(\mathrm{~s}, 3 \mathrm{H}, \mathrm{CH}_{3}\right), 4.74\left(\mathrm{q}, 4 \mathrm{H},-\mathrm{CH}_{2} \mathrm{CH}-3\right), 5.29\left(\mathrm{~d}, 2 \mathrm{H}, \mathrm{CH}_{2}-\mathrm{Ar}\right), 7.62(\mathrm{~d}, \mathrm{H}, \\
\mathrm{C}-\mathrm{O}-\mathrm{CH}), 715(\mathrm{~d}, 2 \mathrm{H}, \mathrm{Ar}-\mathrm{CH}) 7.25-7.40(\mathrm{~m}, 8 \mathrm{H}, \mathrm{Ar}-\mathrm{CH}), 7.93(\mathrm{~d}, 1 \mathrm{H}, \\
\mathrm{CH}=\mathrm{CH}), 8.20(\mathrm{t}, 3 \mathrm{H}, \mathrm{Ar}-\mathrm{CH}) \mathrm{ppm} .\end{array}$ \\
\hline
\end{tabular}




\begin{tabular}{|c|c|c|c|}
\hline 12. & E-4 & $\begin{array}{l}3441,3057(\mathrm{CH} \text { str, } \\
\mathrm{Ar}), 2971\left(\mathrm{CH}_{2} \mathrm{CH}_{3}\right), 2932,1654(\mathrm{C}=0), 1084,623 \\
(\mathrm{C}-\mathrm{Cl}), 592(\mathrm{C}=\mathrm{N})\end{array}$ & $\begin{array}{l}1.51\left(\mathrm{t}, 3 \mathrm{H}, \mathrm{CH}_{3}\right), 4.76\left(\mathrm{q}, 2 \mathrm{H},-\mathrm{CH}_{2} \mathrm{CH}_{3}\right), 5.44\left(\mathrm{~d}, 2 \mathrm{H}, \mathrm{CH}_{2}-\mathrm{Ar}\right), 7.60 \\
(\mathrm{~d}, \mathrm{H}, \mathrm{C}-\mathrm{O}-\mathrm{CH}), 7.23-7.38(\mathrm{~m}, 8 \mathrm{H}, \mathrm{Ar}-\mathrm{CH}), 7.92(\mathrm{~d}, 1 \mathrm{H}, \mathrm{CH}=\mathrm{CH}) \\
\text { 8.1-8.35(m, 4H,Ar)ppm. }\end{array}$ \\
\hline 13. & CL-1 & $\begin{array}{l}3173(\mathrm{~N}-\mathrm{H} \text { str),3059, 2973, 2922,1775(CH-str), } \\
1651(\mathrm{C}=0), 1570(\mathrm{C}=\mathrm{C}), 737(\mathrm{C}-\mathrm{Cl})\end{array}$ & $\begin{array}{l}\left.\text { 6.05(s, 2H, } \mathrm{CH}_{2}-\mathrm{Ar}\right), 6.40(\mathrm{~d}, 1 \mathrm{H}, \mathrm{C}-\mathrm{O}-\mathrm{CH}), 7.08(\mathrm{~d}, 1 \mathrm{H}, \mathrm{CH}=\mathrm{CH}) 7.25-7.37 \\
(\mathrm{~m}, 8 \mathrm{H}, \mathrm{Ar}) 7.94(\mathrm{~m}, 4 \mathrm{H}, \mathrm{Ar}-\mathrm{CH}), 11.91(\mathrm{br}, 1 \mathrm{H}, \mathrm{NH}) .\end{array}$ \\
\hline 14. & CL-2 & $\begin{array}{l}\text { 3093,3059(CH str, Ar), } 1647(\mathrm{C}=0), 1525(\mathrm{C}-\mathrm{H} \\
\text { Str),5 733.5(C-Cl). }\end{array}$ & $\begin{array}{l}1.47\left(\mathrm{t}, 3 \mathrm{H}, \mathrm{CH}_{3}\right) 4.23\left(\mathrm{~m}, 2 \mathrm{H}, \mathrm{CH}_{2}\right) 6.06\left(\mathrm{~s}, 2 \mathrm{H}, \mathrm{Ar}-\mathrm{CH}_{2}\right), 6.42(\mathrm{~d}, 1 \mathrm{H}, \mathrm{C}-\mathrm{O}-\mathrm{CH} \\
7.13(\mathrm{~d}, 1 \mathrm{H}, \mathrm{CH}=\mathrm{CH}),), 7.23-7.54(\mathrm{~m}, 8 \mathrm{H}, \mathrm{Ar}-\mathrm{CH}), 8.07(\mathrm{~m}, 4 \mathrm{H}, \mathrm{Ar}-\mathrm{CH})\end{array}$ \\
\hline 15. & CL-3 & $\begin{array}{l}\text { 3054(CH str, Ar), 3029, } 1656(\mathrm{C}=0) \\
1569(\mathrm{CH}=\mathrm{CH}, \mathrm{Str}), 739(\mathrm{C}-\mathrm{Cl})\end{array}$ & $\begin{array}{l}\left.\left.\left.\text { 5.51(s, 2H, } \mathrm{CH}_{2}-\mathrm{Ar}\right), 5.54\left(\mathrm{~s}, 2 \mathrm{H}, \mathrm{CH}_{2}-\mathrm{Ar}\right) 6.75(\mathrm{~d}, 1 \mathrm{H}, \mathrm{C}-\mathrm{O}-\mathrm{CH}),\right)\right] 7.41(\mathrm{~d}, \\
1 \mathrm{H}, \mathrm{CH}=\mathrm{CH}), 7.23-7.34(\mathrm{~m}, 12 \mathrm{H}, \mathrm{Ar}-\mathrm{CH}), 8.11(\mathrm{~m}, 5 \mathrm{H}, \mathrm{Ar}-\mathrm{CH}) .\end{array}$ \\
\hline 16. & CL-4 & $\begin{array}{l}\text { 3058(CH str, Ar), } 1643(\mathrm{C}=0), 1522(\mathrm{C}-\mathrm{H} \mathrm{Str}) \text {, } \\
\text { 738(C-Cl) }\end{array}$ & $\begin{array}{l}5.58\left(\mathrm{~s}, 2 \mathrm{H}, \mathrm{CH}_{2}-\mathrm{Ar}\right), 6.08\left(\mathrm{~s}, 2 \mathrm{H}, \mathrm{CH}_{2}-\mathrm{Ar}\right), 6.39(\mathrm{~d}, 1 \mathrm{H}, \mathrm{C}-\mathrm{O}-\mathrm{CH}), 6.89(\mathrm{~d}, 1 \mathrm{H}, \\
\mathrm{CH}=\mathrm{CH}), 7.13-7.28(\mathrm{~m}, 13 \mathrm{H}, \mathrm{Ar}-\mathrm{CH}), 7.96(\mathrm{~m}, 4 \mathrm{H}, \mathrm{Ar}-\mathrm{CH})\end{array}$ \\
\hline 17. & A-1 & $\begin{array}{l}3254(\mathrm{~N}-\mathrm{H} \text { str }), 3063,2931(\mathrm{CH} \text { str, Ar), } \\
1649(\mathrm{C}=0), 1525(\mathrm{C}=\mathrm{C} \text { Str })\end{array}$ & $\begin{array}{l}3.63\left(\mathrm{~d}, 2 \mathrm{H}, \mathrm{CH}_{2}\right), 4.91\left(\mathrm{~d}, 2 \mathrm{H}, \mathrm{N}-\mathrm{CH}_{2}\right), 5.24\left(\mathrm{~m}, 1 \mathrm{H}, \mathrm{CH}=\mathrm{CH}_{2}\right), 6.89(\mathrm{~d}, 1 \mathrm{H}, \\
\mathrm{C}-\mathrm{O}-\mathrm{CH}), 7.13-8.19(\mathrm{~m}, 8 \mathrm{H}, \mathrm{Ar}-\mathrm{CH}) 7.77(\mathrm{~d}, 1 \mathrm{H}, \mathrm{CH}=\mathrm{CH}), 11.91(\mathrm{br}, 1 \mathrm{H}, \\
\mathrm{NH}) \mathrm{ppm} \text {. }\end{array}$ \\
\hline 18. & A-2 & $\begin{array}{l}3294,3092,3052,2980,(\mathrm{CH} \text { str, } \mathrm{Ar}) 1650(\mathrm{C}=0) \\
1527(\mathrm{C}=\mathrm{C} \text { Str }), 1447\left(\mathrm{~N}-\mathrm{CH}_{2}\right)\end{array}$ & $\begin{array}{l}1.49\left(\mathrm{~s}, 3 \mathrm{H}, \mathrm{CH}_{3}\right) 5 \cdot 28\left(\mathrm{~d}, 2 \mathrm{H}, \mathrm{NCH}_{2}\right), 5.98\left(\mathrm{~m}, 1 \mathrm{H}, \mathrm{CH}=\mathrm{CH}_{2}\right), 5 \cdot 14(\mathrm{~s}, 2 \mathrm{H}, \\
\left.\mathrm{CH}_{2}\right), 7.63(\mathrm{~d}, \mathrm{H}, \mathrm{C}-\mathrm{O}-\mathrm{CH}) 7.94(\mathrm{~d}, 1 \mathrm{H}, \mathrm{CH}=\mathrm{CH}), 7.22-7.99(\mathrm{~m}, 8 \mathrm{H}, \mathrm{Ar}-\mathrm{CH}), \\
8.13\left(\mathrm{q}, 2 \mathrm{H}, \mathrm{CH}_{2}\right) \text { ppm. }\end{array}$ \\
\hline 19. & A-3 & $\begin{array}{l}\text { 3091, 3026, 3054, 2985(CH str, Ar), } \\
\text { 1644(C=0), 1524(CH=CH Str), }\end{array}$ & $\begin{array}{l}4.7\left(\mathrm{~s}, 2 \mathrm{H}, \mathrm{Ar}-\mathrm{CH}_{2}\right), 5.18\left(\mathrm{~m}, 1 \mathrm{H},=\mathrm{CH}_{2}\right), 5 \cdot 26\left(\mathrm{~d}, 1 \mathrm{H},=\mathrm{CH}_{2}\right) 5 \cdot 84(\mathrm{~d}, 2 \mathrm{H}, \\
\left.\mathrm{NCH}_{2}\right), 7.77(\mathrm{~d}, \mathrm{H}, \mathrm{C}-\mathrm{O}-\mathrm{CH}), 8.02(\mathrm{~d}, 1 \mathrm{H}, \mathrm{CH}=\mathrm{CH}), 7.23-8.30(\mathrm{~m}, 13 \mathrm{H}, \mathrm{Ar}- \\
\mathrm{CH}), 8.12\left(\mathrm{~s}, 2 \mathrm{H}, \mathrm{CH}_{2}\right) \mathrm{ppm} .\end{array}$ \\
\hline 20. & A-4 & $\begin{array}{l}3107,3058(\mathrm{CH} \text { str, } \mathrm{Ar}), 1768(\mathrm{C}=\mathrm{CH}-\mathrm{C}) \\
1650(\mathrm{C}=0), 1527(\mathrm{CH}=\mathrm{CH}), 741(\mathrm{C}-\mathrm{Cl})\end{array}$ & $\begin{array}{l}4.7\left(\mathrm{~s}, 2 \mathrm{H}, \mathrm{CH}_{2}\right), 5.95\left(\mathrm{~m}, 1 \mathrm{H}, \mathrm{CH}=\mathrm{CH}_{2}\right), 5 \cdot 14\left(\mathrm{~d}, 2 \mathrm{H}, \mathrm{NCH}_{2}\right), 5 \cdot 18(\mathrm{~d}, 1 \mathrm{H}, \\
\left.=\mathrm{CH}_{2}\right), 6.49(\mathrm{~d}, \mathrm{H}, \mathrm{C}-\mathrm{O}-\mathrm{CH}), 7.00-7.69(\mathrm{~m}, 12 \mathrm{H}, \mathrm{Ar}-\mathrm{CH}) 7.98(\mathrm{~d}, 1 \mathrm{H}, \\
\mathrm{CH}=\mathrm{CH}) \mathrm{ppm} .\end{array}$ \\
\hline 21. & A-5 & $\begin{array}{l}3399,3053(\mathrm{CH} \text { str, } \mathrm{Ar}), 2958 \\
2871,\left(\mathrm{CH}_{3} \mathrm{CH}_{2} \text { str }\right) 1687,1651(\mathrm{C}=0) \\
1525(\mathrm{CH}=\mathrm{CH} \text { Str })\end{array}$ & $\begin{array}{l}0.92\left(\mathrm{~s}, 6 \mathrm{H}, \mathrm{CH}_{3}-\mathrm{CH}_{3}\right), 2.30\left(\mathrm{~m}, 1 \mathrm{H}, \mathrm{CH}=\mathrm{CH}_{2}\right), 4.53\left(\mathrm{~s}, 2 \mathrm{H}, \mathrm{CH}_{2}\right), \\
4.55\left(\mathrm{~s}, 2 \mathrm{H}, \mathrm{CH}_{2}\right), 5.95\left(\mathrm{~m}, 1 \mathrm{H}, \mathrm{CH}=\mathrm{CH}_{2}\right), 5 \cdot 14\left(\mathrm{~d}, 2 \mathrm{H}, \mathrm{NCH}_{2}\right), 7.61(\mathrm{~d}, \mathrm{H}, \mathrm{C}- \\
\text { O-CH),7.25-7.46(m, } 6 \mathrm{H}, \mathrm{Ar}-\mathrm{CH}), 7.98(\mathrm{~d}, 1 \mathrm{H}, \mathrm{CH}=\mathrm{CH}), 8.12-8.25(\mathrm{t}, 2 \mathrm{H}, \\
\text { Ar)ppm. }\end{array}$ \\
\hline 22. & M-1 & $\begin{array}{l}3390 \text { (N-H str), 3213, 3062(CH str, Ar), 2937, } \\
1647 \text { (C=0), 1566, 1521 (CH=CH Str). }\end{array}$ & $\begin{array}{l}3.65\left(\mathrm{~s}, 3 \mathrm{H}, \mathrm{CH}_{3}\right), 7.34-7.53(\mathrm{~m}, 4 \mathrm{H}, \mathrm{Ar}-\mathrm{CH}), 7.74(\mathrm{~d}, \mathrm{H}, \mathrm{C}-\mathrm{O}-\mathrm{CH}) 8.02(\mathrm{~d}, \\
2 \mathrm{H}, \mathrm{Ar}), 8.10(\mathrm{t}, 3 \mathrm{H}, \mathrm{Ar}), 8.25(\mathrm{~d}, 1 \mathrm{H}, \mathrm{CH}=\mathrm{CH}) 13.18(\mathrm{br}, 1 \mathrm{H}, \mathrm{NH}) \mathrm{ppm} .\end{array}$ \\
\hline 23. & M-2 & $\begin{array}{l}3430,3215,3047(\mathrm{CH} \text { str, } \mathrm{Ar}), 3101,1648(\mathrm{C}=0) \\
1528(\mathrm{CH}=\mathrm{CH} \text { str }), 1462\left(\mathrm{~N}-\mathrm{CH}_{2}\right), 592(\mathrm{C}=\mathrm{CH})\end{array}$ & $\begin{array}{l}1.44\left(\mathrm{t}, 3 \mathrm{H}_{1} \mathrm{CH}_{3}\right), 3.53\left(\mathrm{~s}, 3 \mathrm{H}, \mathrm{CH}_{3}\right), 4.77\left(\mathrm{~d}, 2 \mathrm{H}, \mathrm{CH}_{2}\right), 7.53-7.55(\mathrm{~m}, 4 \mathrm{H}, \mathrm{Ar}- \\
\mathrm{CH}), 7.66(\mathrm{~d}, 1 \mathrm{H}, \mathrm{C}-\mathrm{O}-\mathrm{CH}), 7.88(\mathrm{~d}, \mathrm{H}, \mathrm{CH}=\mathrm{CH}), 8.01-8.11(\mathrm{~m}, 4 \mathrm{H}, \mathrm{Ar}-\mathrm{CH}) \\
\text { ppm. }\end{array}$ \\
\hline 24. & M-3 & $\begin{array}{l}\text { 3093, 3055(CH str, Ar),2931(R-CH } 3, \text { str }) \text {, } \\
1647(\mathrm{C}=0), 1527(\mathrm{CH}=\mathrm{CH} \mathrm{Str})\end{array}$ & $\begin{array}{l}3.89\left(\mathrm{~s}, 3 \mathrm{H}, \mathrm{CH}_{3}\right), 7.31-7.48(\mathrm{~m}, 8 \mathrm{H}, \mathrm{Ar}-\mathrm{CH}), 7.59(\mathrm{~d}, 1 \mathrm{H}, \mathrm{C}-\mathrm{O}-\mathrm{CH}), 7.93(\mathrm{~d}, \\
\mathrm{H}, \mathrm{CH}=\mathrm{CH}), 7.95-8.01(\mathrm{~m}, 3 \mathrm{H}, \mathrm{Ar}-\mathrm{CH}), 8.11(\mathrm{~d}, 2 \mathrm{H}, \mathrm{Ar}-\mathrm{CH}) \mathrm{ppm} .\end{array}$ \\
\hline 25. & M-4 & $\begin{array}{l}\text { 3402, 3202, 3286, 3096(CH str, Ar), 3055, } \\
\text { 2987(R-CH }), 1646(\mathrm{C}=0), 1527(\mathrm{CH}=\mathrm{CH} \mathrm{Str}) \\
1441(\mathrm{~N}-\mathrm{CH} 2), 1064,633(\mathrm{C}-\mathrm{Cl})\end{array}$ & $\begin{array}{l}3.89\left(\mathrm{~s}, 3 \mathrm{H}, \mathrm{CH}_{3}\right), 6.08\left(\mathrm{~s}, 2 \mathrm{H}, \mathrm{CH}_{2}\right), 6.38(\mathrm{~d}, \mathrm{H}, \mathrm{C}-\mathrm{O}-\mathrm{CH}), 7.24(\mathrm{~d}, 1 \mathrm{H}, \\
\mathrm{CH}=\mathrm{CH}), 7.35-7.49(\mathrm{~m}, 8 \mathrm{H}, \mathrm{Ar}-\mathrm{CH}), 7.94-8.13(\mathrm{~m}, 4 \mathrm{H}, \mathrm{Ar}-\mathrm{CH}) \mathrm{ppm} .\end{array}$ \\
\hline 26. & M-5 & $\begin{array}{l}\text { 3104, 3053(CH str, Ar), 2934, 1643(C=0), } \\
\text { 1524(CH-CH Str). }\end{array}$ & $\begin{array}{l}; 0.90\left(\mathrm{~s}, 6 \mathrm{H}, \mathrm{CH}_{3}-\mathrm{CH}_{3}\right), 1.6\left(\mathrm{~s}, 3 \mathrm{H}, \mathrm{CH}_{3}\right), 2.21\left(\mathrm{~m}, 1 \mathrm{H}, \mathrm{CH}=\mathrm{CH}_{2}\right), 4.57(\mathrm{q} \\
\left.2 \mathrm{H}, \mathrm{CH}_{2}\right), 7.68(\mathrm{~d}, 1 \mathrm{H}, \mathrm{C}-\mathrm{O}-\mathrm{CH}), 7.87(\mathrm{~d}, \mathrm{H}, \mathrm{CH}=\mathrm{CH}), 7.31-7.36(\mathrm{~m}, 4 \mathrm{H}, \mathrm{Ar}- \\
\mathrm{CH}), 8.02-8.11(\mathrm{~m}, 3 \mathrm{H}, \mathrm{Ar}-\mathrm{CH}) \mathrm{ppm} .\end{array}$ \\
\hline 27. & $\mathrm{I}-1$ & $\begin{array}{l}3176(\mathrm{NH}-\mathrm{Str}), 2955,1898(\mathrm{CH} \text { str, Ar), } \\
1634(\mathrm{C}=0), 1519(\mathrm{CH}=\mathrm{CH} \mathrm{Str}) .\end{array}$ & $\begin{array}{l}1.35\left(\mathrm{~s}, 6 \mathrm{H}, \mathrm{CH}_{3}-\mathrm{CH}_{3}\right), 2.27\left(\mathrm{~m}, 1 \mathrm{H}, \mathrm{CH}=\mathrm{CH}_{2}\right), 4.57\left(\mathrm{~d}, 2 \mathrm{H}, \mathrm{CH}_{2}\right), 7.61(\mathrm{~d}, \mathrm{H}, \mathrm{C}- \\
\mathrm{O}-\mathrm{CH}), 7.87(\mathrm{~d}, 1 \mathrm{H}, \mathrm{CH}=\mathrm{CH}) 7.18-7.65(\mathrm{~m}, 5 \mathrm{H}, \mathrm{Ar}-\mathrm{CH}), 7.96-8.16(\mathrm{~m}, 4 \mathrm{H}, \\
\text { Ar-CH),11.86(br, } 1 \mathrm{H}, \mathrm{NH}) \mathrm{ppm} .\end{array}$ \\
\hline 28. & $\mathrm{I}-2$ & $\begin{array}{l}3368,3107,3053(\mathrm{CH} \text { str, } \\
\mathrm{Ar}), 1642(\mathrm{C}=0), 1522(\mathrm{CH}=\mathrm{CH} \text { str })\end{array}$ & $\begin{array}{l}0.92\left(\mathrm{~s}, 6 \mathrm{H}, \mathrm{CH}_{3}-\mathrm{CH}_{3}\right), 1.50\left(\mathrm{t}, 3 \mathrm{H}, \mathrm{CH}_{3}-\mathrm{CH}_{2}\right), 2.36\left(\mathrm{~m}, 1 \mathrm{H}, \mathrm{CH}=\mathrm{CH}_{2}\right), 4.20 \\
\left(\mathrm{q}, 2 \mathrm{H}, \mathrm{CH}_{2}\right), 4.56\left(\mathrm{~d}, 2 \mathrm{H}, \mathrm{CH}_{2}\right), 7.46(\mathrm{~d}, \mathrm{H}, \mathrm{C}-\mathrm{O}-\mathrm{CH}), 7.25-7.40(\mathrm{~m}, 5 \mathrm{H}, \mathrm{Ar}- \\
\mathrm{CH}), 7.95(\mathrm{~d}, 1 \mathrm{H}, \mathrm{CH}=\mathrm{CH}), 8.11-8.21(\mathrm{~m}, 3 \mathrm{H}, \mathrm{Ar}-\mathrm{CH}) \mathrm{ppm} .\end{array}$ \\
\hline 29. & I-3 & $\begin{array}{l}\text { 3083,3056, } 3029 \text { (Ar-CH str), } 1646(\mathrm{C}=0) \text {, } \\
\text { 1526(CH=CH Str). }\end{array}$ & $\begin{array}{l}0.65\left(\mathrm{~d}, 6 \mathrm{H}, \mathrm{CH}_{3}-\mathrm{CH}_{3}\right), 2.02\left(\mathrm{~m}, 1 \mathrm{H}, \mathrm{CH}=\mathrm{CH}_{2}\right), 4.39\left(\mathrm{~d}, 2 \mathrm{H}, \mathrm{CH}_{2}\right), 5.3(\mathrm{~s}, 2 \mathrm{H}, \\
\left.\mathrm{CH}_{2}-\mathrm{Ar}\right), 7.09-7.19(\mathrm{~m}, 9 \mathrm{H}, \mathrm{Ar}-\mathrm{CH}), 7.27(\mathrm{~d}, 1 \mathrm{H}, \mathrm{C}-\mathrm{O}-\mathrm{CH}), 7.72(\mathrm{~d}, 1 \mathrm{H}, \\
\mathrm{CH}=\mathrm{CH}), 7.77(\mathrm{~d}, 1 \mathrm{H}, \mathrm{Ar}-\mathrm{CH}), 7.97-8.22(\mathrm{t}, 3 \mathrm{H}, \mathrm{Ar}-\mathrm{CH}) \mathrm{ppm} .\end{array}$ \\
\hline 30. & $\mathrm{I}-4$ & $\begin{array}{l}3294,3090(\mathrm{CH}=\mathrm{CHAr}), 1650(\mathrm{C}=0) \\
1525(\mathrm{CH}=\mathrm{CH} \text { str }), 625(\mathrm{C}-\mathrm{Cl})\end{array}$ & $\begin{array}{l}0.73\left(\mathrm{~d}, 6 \mathrm{H}, \mathrm{CH}_{3}-\mathrm{CH}_{3}\right), 2.05\left(\mathrm{~m}, 1 \mathrm{H}, \mathrm{CH}=\mathrm{CH}_{2}\right), 4.27\left(\mathrm{~d}, 1 \mathrm{H}, \mathrm{CH}_{2}\right), 5.47(\mathrm{~s}, 2 \mathrm{H}, \\
\left.\mathrm{CH}_{2}\right), 6.77(\mathrm{~d}, 1 \mathrm{H}, \mathrm{C}-\mathrm{O}-\mathrm{CH}), 7.10-7.40(\mathrm{~m}, 8 \mathrm{H}, \mathrm{Ar}-\mathrm{CH}), 7.77(\mathrm{~d}, 1 \mathrm{H}, \mathrm{CH}=\mathrm{CH}), \\
7.80-8.21(\mathrm{~m}, 4 \mathrm{H}, \mathrm{Ar}-\mathrm{CH}) \mathrm{ppm} .\end{array}$ \\
\hline
\end{tabular}

Table 3: The \% inhibitory effect of antiangiogenic activity $\mathrm{N}-1$ substituted indolylchalconehybrids and in a CAM assay in a concentration of $0.1 \mu \mathrm{M} / \mathrm{egg}$

\begin{tabular}{|c|c|c|c|c|c|c|c|c|}
\hline $\begin{array}{l}\text { Code of } \\
\text { compound }\end{array}$ & $\begin{array}{l}\text { No\% } \\
\text { avascular } \\
\text { CAM/Total } \\
\mathrm{N}=6\end{array}$ & $\begin{array}{l}\text { Std. error } \\
\text { of } \\
\text { deviation }\end{array}$ & $\begin{array}{l}\text { Code of } \\
\text { compound }\end{array}$ & $\begin{array}{l}\text { No \% } \\
\text { avascular } \\
\text { CAM/Total } \\
\mathrm{N}=6\end{array}$ & $\begin{array}{l}\text { Std. error } \\
\text { of } \\
\text { deviation }\end{array}$ & $\begin{array}{l}\text { Code of } \\
\text { compound }\end{array}$ & $\begin{array}{l}\text { No\% } \\
\text { avascular } \\
\text { CAM/Total } \\
\mathrm{N}=6\end{array}$ & $\begin{array}{l}\text { Std. error } \\
\text { of } \\
\text { deviation }\end{array}$ \\
\hline B-1 & $14 / 20(70 \%)$ & 1.826 & CL-3 & $2 / 20(10 \%)$ & 0.5774 & A-1 & $6 / 20(30 \%)$ & 1.390 \\
\hline B-2 & $13 / 20(65 \%)$ & 1.390 & CL-4 & $7 / 20(35 \%)$ & 1.390 & A-2 & $5 / 20(25 \%)$ & 0.5774 \\
\hline B-3 & $8 / 20(40 \%)$ & 1.390 & E-1 & $18 / 20(90 \%)$ & 0.5774 & A-3 & $4 / 20(20 \%)$ & 1.390 \\
\hline B-4 & $7 / 20(35 \%)$ & 1.390 & E-2 & $19 / 20(95 \%)$ & 0.2582 & A-4 & $3 / 20(15 \%)$ & 13.336 \\
\hline C-1 & $18 / 20(90 \%)$ & 0.5774 & E-3 & $15 / 20(75 \%)$ & 1.155 & $A-5$ & $14 / 20(70 \%)$ & 0.8165 \\
\hline $\mathrm{C}-2$ & $17 / 20(85 \%)$ & 1.317 & E-4 & $6 / 20(30 \%)$ & 1.390 & M-1 & $15 / 20(75 \%)$ & 1.155 \\
\hline C-3 & $16 / 20(80 \%)$ & 0.9309 & $\mathrm{I}-1$ & $16 / 20(80 \%)$ & 0.9309 & M-2 & $8 / 20(40 \%)$ & 6.731 \\
\hline$C-4$ & $15 / 20(75 \%)$ & 1.155 & $\mathrm{I}-2$ & $18 / 20(90 \%)$ & 0.8165 & M-3 & $7 / 20(35 \%)$ & 0.8165 \\
\hline CL-1 & $17 / 20(85 \%)$ & 1.317 & $\mathrm{I}-3$ & $7 / 20(35 \%)$ & 0.8165 & M-4 & $6 / 20(30 \%)$ & 1.390 \\
\hline CL-2 & $5 / 20(25 \%)$ & 1.317 & $\mathrm{I}-4$ & $6 / 20(30 \%)$ & 1.390 & M-5 & $16 / 20(80 \%)$ & 0.7303 \\
\hline
\end{tabular}

*SD $(\mathrm{n}=6)$ 


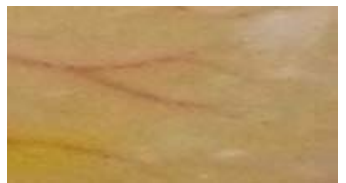

(a) C-2 at dose $2.5 \mu \mathrm{M}$

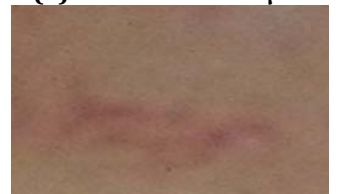

(d) I-2at dose $2.5 \mu \mathrm{M}$

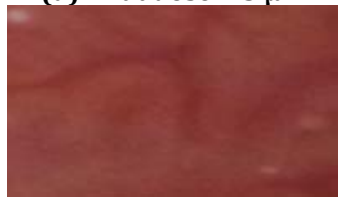

(g)-Ve Control

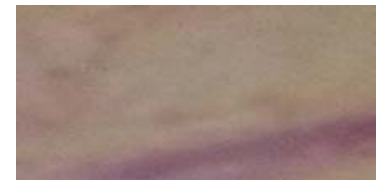

(b) C-2at dose $5 \mu \mathrm{M}$

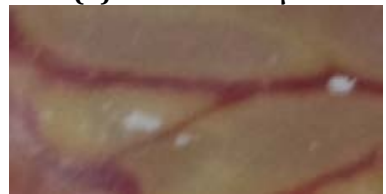

(e) I-2at dose $5 \mu \mathrm{M}$

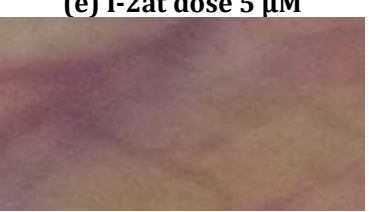

(h) DMSO

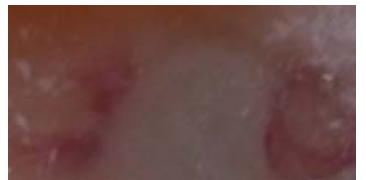

(c) C-2at dose10 $\mu \mathrm{M}$

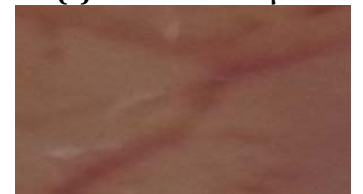

(f) I-2at dose10 $\mu \mathrm{M}$

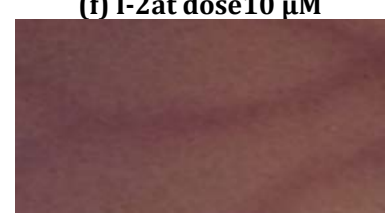

(i)+Ve Control

Fig. 1A: Effect of dose-dependent assay of synthesized indolylchalcone hybrids of benzimidazole on in vivo CAM angiogenesis: CAM were treated with different series of dose such as $2.5 \mu \mathrm{M}, 5 \mu \mathrm{M}$ and $10 \mu \mathrm{M}$ treatment groups indolyl chalcone hybrids at day $8^{\text {th }}$, the percentage of inhibition of blood vesel formation, compared to untreated conrol, was determined. (a to i) Result suggests that indolyl chalcone hybrids compound code $\mathrm{C}-2$ and $\mathrm{I}-2$ inhibited angiogenesis expressed as ( $\mathrm{p}=<0.001$ vs DMSO)

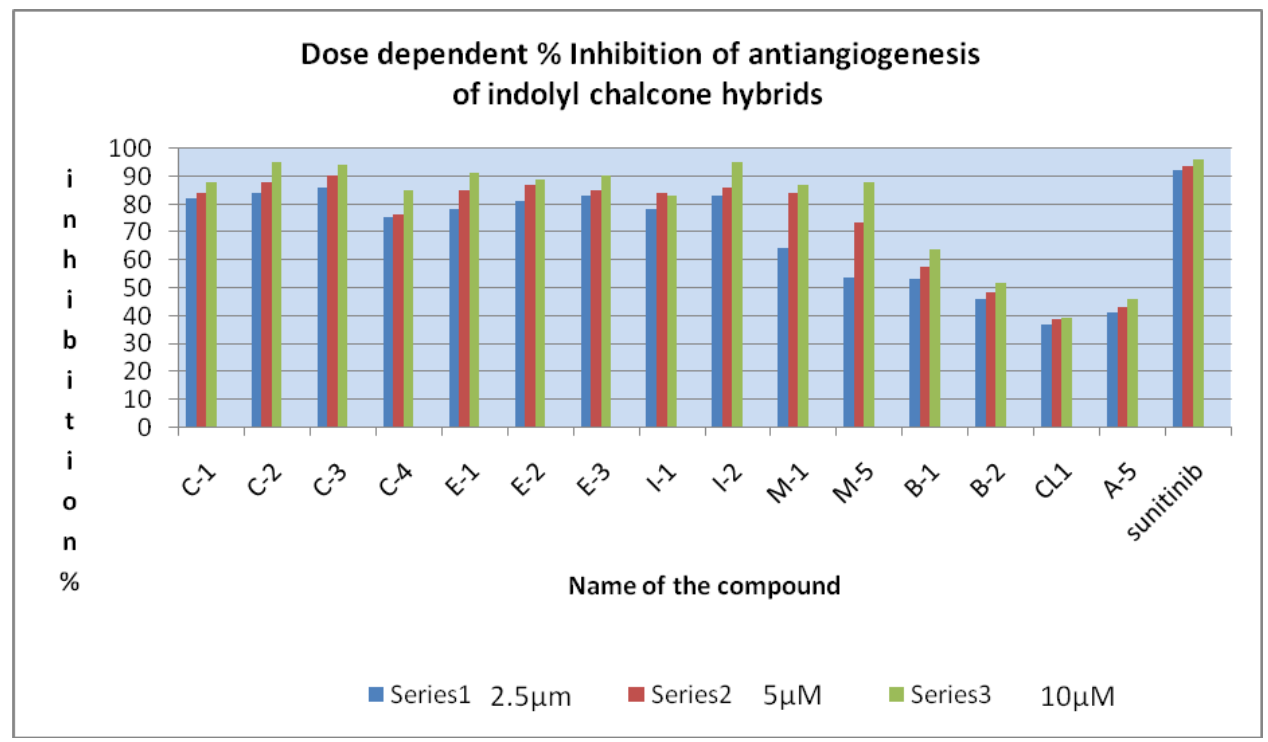

Fig. 1A: Effect of dose-dependent assay of synthesized indolylchalcone hybrids of benzimidazole on in vivo CAM angiogenesis

\section{DISCUSSION}

We found during our CAM Assay Study that there was noticeable decrease in number of blood vessels as compared with the negative control group, as shown in fig. 1A. Furthermore, One-way ANOVA was used to compare the means of groups. Results showed that the different series of dose such as $2.5 \mu \mathrm{M}, 5 \mu \mathrm{M}$ and $10 \mu \mathrm{M}$ treatment groups exhibited varying response based on their \%inhibition on blood vessel count (fig. 1B). These newly synthesized indolylchalcone hybrids were statistically significant at $\mathrm{P}=0.05$. Considering this we can draw an inference that amongst 30 synthesized compounds 11 compounds of indolylchalcone hybrids has potent anti-angiogenic properties.

\section{CONCLUSION}

We have developed an inexpensive, simple, and eco-friendly synthesis of $\mathrm{N}$-alkyl derivatives of two fused heterocyclic compounds of indole-3-carboxaldehyde and 2-acetylbenzimidazole. The 11 compounds of indolylchalcones as shown in graph possess very good dose-dependent anti-angiogenic activities. The $\mathrm{N}-1$ substitution is valid on benzimidazole such as ethyl, benzyl and also 2 - $\mathrm{Cl}$ substituted group on phenyl shows significant potent anticancer activity. The potency of anti-angiogenetic activity shows that methyl>Ethyl $>$ Cl-benzyl>Benzyl>Isobutyl.

The present study suggests that N-1 substituted Indolylchalcone hybrids of 2-acetyl benzimidazole might be promising analogs of angiogenesis inhibitors to manage the uncontrolled neovascularization occurring during malignant tumor development.

\section{ACKNOWLEDGEMENT}

Authors take this opportunity to thank UGC-BSR for funding this project. Authors also thankful to the Director of SAIF of Punjab University, Chandigarh for recording IR-spectra and NMR-spectra of synthesized compounds. 


\section{AUTHORS CONTRIBUTIONS}

All the authors have contributed equally to this study.

\section{CONFLICT OF INTERESTS}

Declared none

\section{REFERENCES}

1. Venkateswaran A, Reddy Y, Sonar V, Muthusamy V, Crooks P, Freeman $\mathrm{M}$, et al. Antiangiogenic properties of substituted (Z)( \pm )-2-(N-benzylindol-3-ylmethylene)quinuclidin-3-ol/one analogs and their derivatives. Bioorg Med Chem Lett 2010;20:7323-6.

2. Huang L, Shih M, Chen H. Synthesis of N2-(substituted benzyl)3-(4-methylphenyl)indazoles as novel anti-angiogenic agents. Bioorganic Med Chem 2006;14:528-36.

3. Nagaraju G, Zhu S, Ko J. Antiangiogenic effects of a novel synthetic curcumin analogue in pancreatic cancer. Cancer Lett 2015;357:557-65.

4. Oklu R, Walker T, Wicky S, Hesketh R. Angiogenesis and current antiangiogenic strategies for the treatment of cancer. J Vasc Interv Radiol 2010;21:1791-805.

5. Wen Hui Yang, Jun Xu, Jian Bing Mu, Jun Xie. Revision of the concept of anti-angiogenesis and its applications in tumor treatment. Chronic Diseases Translational Med 2017;3:33-40.

6. Tahergorabi Z, Khazaei M. A review on angiogenesis and its assays. Iran J Basic Med Sci 2012;15:1110-26.

7. Gotink K, Verheul H. Anti-angiogenic tyrosine kinase inhibitors: what is their mechanism of action. Angiogenesis 2010;13:1-14.

8. Saraswati S, Agrawal S. Brucine, an indole alkaloid from strychnos nux-vomica, attenuates VEGF-induced angiogenesis via inhibiting VEGFR2 signaling pathway in vitro and in vivo. Cancer Lett 2013;332:83-93.

9. Lee H, Banskota S, Kim D, Been J, Jin Y, Gautam J, et al. Synthesis and antiangiogenic activity of 6-amido-2,4,5-trimethylpyridin3-ols. Bioorganic Med Chem Lett 2014;24:3131-6.
10. He H, Wang X, Shi L, Yin W, Yang Z, Liang Y. Synthesis, antitumor activity and mechanism of action of novel 1,3 thiazole derivatives containing hydrazide-hydrazone and carboxamide moiety. Bioorg Med Chem Lett 2016;14:3263-70.

11. Christodoulou M, Liekens S, Kasiotis K, Haroutounian S. Novel pyrazole derivatives: synthesis and evaluation of antiangiogenic activity. Bioorg Med Chem 2010;18:4338-50.

12. Roopashree R, Mohan C, Swaroop T, Jagadish S, Rangappa K Synthesis, characterization, and in vivo biological evaluation of novel benzimidazoles as potential anticancer agents. Asian J Pharm Clin Res 2014;7:309-13.

13. Nagarajan S, Majumder S, Sharma U, Rajendran S, Kumar N, Chatterjee $\mathrm{S}$, et al. Synthesis and anti-angiogenic activity of benzothiazole, benzimidazole containing phthalimide derivatives. Bioorganic Med Chem Lett 2013;23:287-90.

14. Kumar D, Kumar N, Akamatsu K, Kusaka E, Harada H, Ito T. Synthesis and biological evaluation of indolyl chalcones as antitumor agents. Bioorganic Med Chem Lett 2010;20:3916-9.

15. Kumar D, Maruthi Kumar N, Tantak M, Ogura M, Kusaka E, Ito T. Synthesis and identification of $\alpha$-cyano bis(indolyl)chalcones as novel anticancer agents. Bioorganic Med Chem Lett 2014;24:5170-4.

16. Shi J, Xiao Z, Ihnat M, Kamat C, Pandit B, Hu Z, et al. Structureactivity relationships studies of the anti-angiogenic activities of linomide. Bioorganic Med Chem Lett 2003;13:1187-9.

17. Melkonian G, Munoz N, Chung J, Tong C, Marr R, Talbot P. Capillary plexus development in the day five to day six chick chorioallantoic membrane is inhibited by cytochalasin D and suramin. J Exp Zool 2002;292:241-54.

18. Pandit R, Anil A, Lali A, Indap M. Evaluation of antiangiogenic activity through tubulin interaction of chloroform fraction of the feather star, Lamprometra palmata palmata. Indian J Mar Sci 2009;38:28-37. 